\title{
Reduced Graphene Oxide Encapsulated N-type Si Nanoparticles as Anode for Lithium-ion Batteries
}

\author{
Zhongwei Luo, Taotao Ding, Jiangnai Dai , Changqing Chen ${ }^{*}$ \\ Wuhan National Laboratory for Optoelectronics, Huazhong University of Science and Technology, \\ Wuhan 430074, China. \\ *E-mail: cqchen@hust.edu.cn, daijiangnan@hust.edu.cn
}

doi: $10.20964 / 2016.06 .4$

Received: 16 February 2016 / Accepted: 22 March 2016 / Published: 4 May 2016

Silicon is generally considered as the next generation of anode materials for lithium-ion batteries because of its highest theoretical specific capacity of all the known materials. However its low initial coulombic efficiency, poor cyclic performance and expensive silicon nanoparticles limit its application for anode. Here, we report a simple and inexpensive synthetic method of RGO/n-Si composite in which $\mathrm{n}$-Si nanoparticles were encapsulate by thermal reduced graphene oxide, in which the thermal reduced garphene oxide buffers the huge volume changes of Si during lithiation and delithiation. Besides, Si nanoparticles doped with $\mathrm{P}$ can improve the electrical conductivity of anode. As a result, these graphene encapsulated n-type Si nanoparticles have a much improved cyclic stability with a reversible capacity of $1529 \mathrm{mAh} / \mathrm{g}$ over 200 cycles.

Keywords: $\mathrm{Si}$; n-Si, graphene, lithium-ion batteries.

\section{$\underline{\text { FULL TEXT }}$}

(C) 2016 The Authors. Published by ESG (www.electrochemsci.org). This article is an open access article distributed under the terms and conditions of the Creative Commons Attribution license (http://creativecommons.org/licenses/by/4.0/). 